Published in final edited form as:

Nat Cell Biol. 2009 November ; 11(11): 1325-1331. doi:10.1038/ncb1976.

\title{
Slit2-Robo4 signalling promotes vascular stability by blocking Arf6 activity
}

Christopher A. Jones ${ }^{1,2,14}$, Naoyuki Nishiya $3,11,14$, Nyall R. London ${ }^{1,2,14}$, Weiquan Zhu $^{1,2}$, Lise K. Sorensen ${ }^{2}$, Aubrey C. Chan ${ }^{1,2}$, Chinten J. Lim ${ }^{3}$, Haoyu Chen 4,11 , Qisheng Zhang $^{5}$, Peter G. Schultz ${ }^{6}$, Alaa M. Hayallah ${ }^{7,12}$, Kirk R. Thomas ${ }^{2,8}$, Michael Famulok $^{7}$, Kang $^{2}$ Zhang ${ }^{4,13}$, Mark H. Ginsberg ${ }^{3,15}$, and Dean Y. Li ${ }^{1,2,9,15}$

${ }^{1}$ Department of Oncological Sciences, University of Utah, Salt Lake City, UT 84112, USA.

${ }^{2}$ Molecular Medicine Program, University of Utah, Salt Lake City, UT 84112, USA.

${ }^{3}$ Department of Medicine, University of California San Diego, La Jolla, CA 92093-0726, USA.

${ }^{4}$ Department of Ophthalmology, University of Utah, Salt Lake City, UT 84112, USA.

${ }^{5}$ School of Pharmacy, University of North Carolina at Chapel Hill, NC 27599, USA.

${ }^{6}$ Department of Chemistry and The Skaggs Institute for Chemical Biology, The Scripps Research Institute, La Jolla, CA 92037, USA.

${ }^{7}$ LIMES Program Unit Chemical Biology \& Medicinal Chemistry, c/o Kekulé Institut für Organische Chemie und Biochemie, University of Bonn, Gerhard-Domagk-Strae 1, 53121 Bonn, Germany.

${ }^{8}$ Division of Haematology, Department of Medicine, University of Utah, Salt Lake City, UT 84112, USA.

${ }^{9}$ Division of Cardiology, Department of Medicine, University of Utah, Salt Lake City, UT 84112, USA.

\section{Abstract}

Slit-Roundabout (Robo) signalling has a well-understood role in axon guidance ${ }^{-}$5. Unlike in the nervous system, however, Slitdependent activation of an endothelial-specific Robo, Robo4, does not initiate a guidance program. Instead, Robo4 maintains the barrier function of the mature vascular

(c) 2009 Macmillan Publishers Limited. All rights reserved.

15 Correspondence should be addressed to D.Y. L. or M.H.G. (dean.li@hmbg.utah.edu; mhginsberg@ucsd.edu) .

${ }_{11}^{11}$ Current address: Joint Shantou International Eye Centre, Shantou University and Chinese University Hong Kong, Shantou, China.

12 Current address: Department of Pharmaceutical Organic Chemistry, Faculty of Pharmacy, Assiut University, Assiut 71526, Egypt.

${ }^{13}$ Current address: University of California at San Diego School of Medicine and VASDHS Centre for Human Genetics and Genomics, La Jolla, CA 92093-0838, USA.

${ }^{14}$ These authors contributed equally to this work

AUTHOR CONTRIBUTIONS C.A.J., N.N., N.R.L., M.H.G. and D.Y.L. were responsible for project conceptualization and planning, experimental design, data analysis, and manuscript preparation.M.H.G. and D.Y.L. were responsible for funding the project. C.A.J., N.N. and N.R.L were responsible for performing all experiments or coordinating experimental design and work of others. W.Z., L.K.S., A.C., C.J.L. and K.R.T. performed specific and necessary experiments presented in the paper or in our response to reviewers. Q.Z., P.G.S., A.M.H., M.F. and K.Z. provided expertise, reagents or assays.

${ }^{10}$ Current address: Department of Microbial Chemical Biology and Drug Discovery, Iwate Medical University School of Pharmaceutical Sciences, 2-1-1 Nishitokuta, Yahaba, Shiwa-gun, Iwate 028-3694, Japan.

Supplementary Information is available on the Nature Cell Biology website.

Reprints and permissions information is available online at http://npg.nature.com/ reprintsandpermissions/.

COMPETING FINANCIAL INTERESTS The authors declare competing financial interests. The University of Utah has filed patents covering the technology described in this manuscript with the intent of commercializing this technology.

METHODS Methods and any associated references are available in the online version of the paper at http://www.nature.com/naturecellbiology/. 
network by inhibiting neovascular tuft formation and endothelial hyperpermeability induced by proangiogenic factors 6 . In this study, we used cell biological and biochemical techniques to elucidate the molecular mechanism underlying the maintenance of vascular stability by Robo4. Here, we demonstrate that Robo4 mediates Slit2-dependent suppression of cellular protrusive activity through direct interaction with the intracellular adaptor protein paxillin and its paralogue, Hic-5. Formation of a Robo4-paxillin complex at the cell surface blocks activation of the small GTPase Arf6 and, consequently, Rac by recruitment of Arf-GAPs (ADP-ribosylation factor- directed GTPaseactivating proteins) such as GIT1. Consistent with these in vitro studies, inhibition of Arf6 activity in vivo phenocopies Robo4 activation by reducing pathologic angiogenesis in choroidal and retinal vascular disease and VEGF-165 (vascular endothelial growth factor-165)-induced retinal hyperpermeability. These data reveal that a Slit2-Robo4-paxillin-GIT1 network inhibits the cellular protrusive activity underlying neovascularization and vascular leak, and identify a new therapeutic target for ameliorating diseases involving the vascular system.

In both developmental and pathologic settings, cells migrate by orienting protrusive events in response to attractive and repulsive cues. Slit2-Robo4 signalling has been shown previously to inhibit migration of cells towards a soluble chemoattractant gradient ${ }^{7,8}$. In addition to chemoattractants, immobilized extracellular matrix proteins, such as fibronectin, have a critical role in regulating cellular motility during development and disease ${ }^{9}$, and gradients of fibronectin direct migration in a process called haptotaxis. We determined whether Slit2Robo4 signalling modulates extracellular matrix-driven motility, using HEK 293 cells expressing fulllength Robo4 or a cytoplasmic tail-domain deletion mutant containing only the ectodomain and the transmembrane domain (Robo4 ED-TM; Fig. 1a). We induced these cells to migrate to fibronectin in the absence (mock) and presence of Slit2, and found that Slit2 inhibited the motility of cells expressing Robo4, but not Robo4 ED-TM (Fig. 1b).

Migrating cells protrude in the forward direction and form new attachments to the extracellular matrix9. These newly formed attachments lead to adhesion-induced activation of the small GTPase Rac, which creates broad protrusive structures called lamellipodia through localized actin polymerization 10 . When detached cells adhere, adhesion-induced Rac activation leads to unpolarized lamellipodial extension, resulting in spreading ${ }^{11}$. As cell spreading is an integral part of migration on the extracellular matrix, we assessed whether Slit2-Robo4 signalling inhibits this process. We found that although cells expressing Robo4 adhered normally to fibronectin and Slit2 (data not shown), they spread significantly less in the presence of Slit2 than cells expressing Robo4 ED-TM or LacZ (Fig. 1c, d).

The inability of Robo4 ED-TM to inhibit spreading showed that the cytoplasmic tail is required for this activity. To test whether it is also sufficient for inhibition of spreading, we generated an $\alpha$ IIb integrin-Robo4 chimaeric protein (Fig. 1a), which enabled initiation of Robo4 signalling with fibrinogen, a ligand for $\alpha$ IIb: $\beta 3$ integrin. Cells expressing $\alpha$ IIb: $\beta 3$ integrin spread equivalently on fibronectin alone, and a mixture of fibronectin and fibrinogen, whereas $\alpha$ IIb integrin-Robo4: $\beta 3$-expressing cells spread significantly less in the presence of fibrinogen (Fig. 1e).

To define the region of the Robo4 cytoplasmic tail that is required for inhibition of fibronectininduced protrusive activity, cells expressing Robo4 deletion constructs (Fig. 1a) were subjected to haptotaxis assays. Migration of cells expressing Robo4 NH2, but not Robo4 COOH, was inhibited by Slit2 (Fig. 1f), indicating the necessity and sufficiency of the amino-terminal half of the Robo4 tail for suppressing cell motility elicited by the extracellular matrix.

Identification of the region of the Robo4 cytoplasmic tail that confers functional activity allowed us to search for cytoplasmic components that might regulate Robo4 signal transduction. Using the N-terminal half of the Robo4 tail as bait, a yeast two-hybrid screen of 
a human aortic cDNA library identified a member of the paxillin family of adaptor proteins, Hic-5, as a potential Robo4-interacting protein (Supplementary Information, Fig. S1).

Hic-5 and its paralogue, paxillin, show cell-type-specific expression ${ }^{12,13}$. Western blotting of CHO-K1, HEK 293 and NIH3T3 cell lysates with antibodies against Hic-5 and paxillin detected paxillin in each cell line, whereas Hic-5 was only found in CHO-K1 and NIH3T3 cells (Supplementary Information, Fig. S1d). As functional studies used HEK 293 cells, this result suggested that paxillin interacts with Robo4 to regulate cell migration. As observed with Hic-5 (Supplementary Information, Fig. S1c), paxillin was identified in Robo4 immunoprecipitates of cells expressing paxillin and the Robo4 cytoplasmic tail, but not paxillin alone (Fig. 2a).

As Slit2 activates Robo4 (refs 7, 8, 14, 15), we asked whether Slit2 stimulation regulates the interaction between Robo4 and paxillin. Full-length Robo4 was immunoprecipitated from cell lysates after incubation in the absence and presence of Slit2, and endogenous paxillin was only detected in Robo4 immunoprecipitates after Slit2 treatment (Fig. 2b).

We defined the region of Robo4 that is required for interaction with paxillin by generating GST-Robo4 fusion proteins spanning the cytoplasmic tail (Supplementary Information, Fig. S2a, b), and performing in vitro binding assays with purified recombinant paxillin (Supplementary Information, Fig. S2c, d). We identified a 70-amino-acid fragment of the Nterminal half of the Robo4 tail as the region that mediates interaction with paxillin (Supplementary Information, Fig. S2d). Deletion of this region prevented interaction with paxillin, as well as the known Robo family-binding protein Mena (murine enabled; data not shown $)^{7}, 16$. As Mena interacts with Robo through the Robo CC2 motif, which lies outside the deleted region, these binding data suggested that elimination of amino acids 604-674 affects conformation of the Robo4 tail.We then created smaller deletions within this 70-amino-acid stretch and performed additional in vitro binding assays (data not shown). Using this approach we identified a mutant GST-Robo4 fusion protein lacking 35 amino acids (604-639) that failed to bind paxillin, but retained interaction with Mena (Fig. 2c). This region of Robo4 is subsequently referred to as the paxillin interaction motif (PIM; Supplementary Information, Fig. S2e). We confirmed the importance of this region in promoting interaction with endogenous paxillin using co-immunoprecipitation from cells expressing wild-type Robo4 cytoplasmic tail or Robo4 $\triangle$ PIM (Fig. 2d).

We next determined whether the PIM is important for receptor activity by performing functional assays with cells expressing Robo4 $\triangle \mathrm{PIM}$. This mutant form of the receptor failed to inhibit fibronectin-induced cell migration and spreading in the presence of Slit2 (Fig. 2e, f), demonstrating that the region of the Robo4 tail necessary for paxillin binding is also required for inhibition of protrusive activity.

Although Robo4 $\triangle$ PIM maintained its interaction with Mena (Fig. 2c), it remained possible that the mutation perturbed interactions between Robo4 and proteins other than paxillin. To investigate this, we generated paxillin mutants that disrupt its association with Robo4. Paxillin is a modular protein composed of $\mathrm{N}$-terminal leucine/aspartic acid (LD) repeats and $\mathrm{C}$-terminal Lim domains ${ }^{17}$ (Supplementary Information, Fig. S2f). Analysis of clones recovered from the yeast two-hybrid screen (Supplementary Information, Fig. S1a) suggested that the Lim domains, particularly Lim3 and Lim4, are important for interaction with Robo4. We performed co-immunoprecipitation experiments using cells expressing the Robo4 tail and either paxillinLD or paxillin-Lim, and found Paxillin-Lim, but not paxillin-LD, was in Robo4 immunoprecipitates (Supplementary Information, Fig. S2g). To clarify which Lim domain is required for binding to Robo4, we made serial deletions from the $\mathrm{C}$ terminus of paxillin, cotransfected these with the Robo4 cytoplasmic tail into cells and performed co- 
immunoprecipitation experiments. Deletion of Lim 4 completely abrogated binding between paxillin and Robo4 (Fig. 2g).

Delineation of the Robo4 binding site on paxillin allowed us to evaluate the role of paxillin in Robo4-dependent processes. If paxillin were required for receptor activity, the paxillin $\Delta \operatorname{Lim} 4$ mutant, which is unable to interact with Robo4, should fail to inhibit fibronectin-induced spreading in the presence of Slit2. Endogenous paxillin was depleted in Robo4-expressing cells using short interfering RNA (siRNA) and then reconstituted with wild-type chicken paxillin (Ch-paxillin) or Ch-paxillin $\Delta$ Lim4, both of which are resistant to siRNA targeting the endogenous gene (Fig. $2 \mathrm{~h}$ ).Cells expressing Ch-paxillin $\Delta$ Lim 4 spread normally in the presence of Slit2, whereas cells expressing Ch-paxillin showed a reduction in cell area characteristic of Slit2-Robo4 signalling (Fig. 2i).

The ability of a cell to spread and migrate on an extracellular matrix protein, such as fibronectin, is regulated by the activation of Rho family GTPases, which include Rho, Cdc42 and Rac ${ }^{18}$. Of these proteins, Rac has an essential role in promoting the actin polymerization that leads to lamellipodial extension and cell spreading during migration ${ }^{18,19}$. To examine whether Slit2Robo4 signalling inhibits adhesion-dependent Rac activation, cells expressing Robo4, Robo $4 \Delta \mathrm{PIM}$ or pcDNA3 alone were plated on fibronectin in the absence and presence of Slit2, and Rac-GTP levels were analysed. Additionally, cells expressing $\alpha$ IIb integrin-Robo4: $\beta 3$ were plated on fibronectin in the absence and presence of fibrinogen, and Rac-GTP levels were analysed. Cells expressing Robo4 or $\alpha$ IIb integrin- Robo4: $\beta 3$ showed significantly less adhesion-stimulated Rac activation when plated on Slit2 and fibrinogen, respectively (Fig. 3a, b). When this experiment was repeated with Robo $4 \Delta$ PIM, we found that cells expressing this mutant receptor were refractory to Slit2 treatment (Fig. 3a).

To confirm that Robo4-dependent inhibition of cell spreading is principally due to suppression of Rac activation, we subjected cells co-expressing Robo4 and a dominant-active form of Rac, $\mathrm{Rac}^{\mathrm{G} 12 \mathrm{~V}}$, tospreading assays. Cells expressing Rac ${ }^{\mathrm{G} 12 \mathrm{~V}}$ were refractory to Slit2 treatment (Fig. 3c), providing additional evidence that Slit2-Robo4 signalling blocks protrusive activity by inhibiting Rac.

We previously demonstrated that the interaction of $\alpha 4$ integrin with a paxillin-GIT1 complex enables $\alpha 4$ integrin to control Rac-dependent changes in protrusive activity by modulating the activity of the small GTPase Arf6 (ref. 20). Our finding that Robo4 interacts with paxillin and inhibits protrusive activity prompted us to determine whether Robo4 signalling impinges on the Arf6 pathway. Cells expressing full-length Robo4 were plated on fibronectin in the absence and presence of Slit2, while those expressing $\alpha$ IIb integrin-Robo4: $\beta 3$ were plated on fibronectin in the absence and presence of fibrinogen, and Arf6-GTP levels were analysed. Whereas fibronectin alone stimulated an increase in Arf6-GTP, both Slit2 and fibrinogen reduced Arf6-GTP levels in cells expressing Robo4 or $\alpha$ IIb integrin-Robo4: $\beta 3$, respectively (Fig. 3d, e).

We analysed the requirement for a paxillin-GIT1 complex in Robo4dependent inhibition of protrusive activity. The GIT1 paxillin-binding sequence (PBS) is at the C terminus of the protein, and ectopic expression of this fragment blocks interaction between GIT1 and paxillin ${ }^{20}$.Cells were transfected with $\alpha$ IIb integrin-Robo4: $\beta 3$ and an empty vector or GIT1PBS, and subjected to spreading assays on fibronectin in the absence and presence of fibrinogen. Cells expressing $\alpha$ IIb integrin-Robo4: $\beta 3$ showed a decrease in cell area when plated on fibronectin and fibrinogen, but this effect was lost in cells expressing GIT1-PBS (Fig. 3f). Similarly, in full-length Robo4-expressing cells plated on fibronectin and Slit2, GIT1-PBS prevented a decrease in cell area (Fig. 3g). 
To determine whether Slit2-Robo4 signalling inhibits protrusive activity by inactivating Arf6, we co-expressed the Arf6 guanine nucleotide exchange factor (GEF) ARNO with Robo4 and performed spreading assays. Overexpression of ARNO blocked the ability of Slit2 to reduce cell area, indicating that a principal effect of Slit2-Robo4 signalling is to prevent GTPloading of Arf6 (Fig. 3g). If ARNO restores the ability of Robo4-expressing cells to spread on fibronectin and Slit2, it should similarly re-establish Rac activation on this matrix. Indeed, overexpression of ARNO led to normal levels of Rac-GTP in cells plated on fibronectin and Slit2 (Fig. 3h).

To assess the effect of Slit2-Robo4 signalling on the subcellular distribution of paxillin, cells were plated on fibronectin in the absence and presence of Slit2, and stained for endogenous paxillin. In the absence of Slit2, cells expressing full-length Robo4 spread normally and formed abundant, paxillin-stained focal adhesions near the cell periphery (Supplementary Information, Fig. S3a, top panel). In the presence of Slit2, however, cells showed reduced spreading, contained less F-actin and formed fewer and smaller paxillin-stained focal adhesions (Supplementary Information, Fig. S3a, bottom panel). Control cells (cells not expressing Robo4) showed similar morphology when adhered on fibronectin alone or on fibronectin and Slit2 (data not shown), indicating that the effect of Slit2 is Robo4-dependent.

We repeated this assay using bovine aortic endothelial(BAE) cells, which endogenously express Robo4. On substrata coated with fibronectin and Slit2, BAE cells showed reduced spreading compared with cells adhered to fibronectin alone (Supplementary Information, Fig. S3b). In addition, BAE cells adhered to fibronectin and Slit2 formed small paxillin-stained structures, which were inconsistent with the mature focal adhesions observed in cells adhered to fibronectin alone (Supplementary Information, Fig. S3b; see arrows). The inhibitory effect of Slit 2 on cell spreading seems to be transient, as cells adhered for extended intervals showed similar morphology and focal adhesion formation irrespective of Slit2 treatment (data not shown).

Our data suggest that in Robo4-expressing cells, Slit2 redistributes paxillin from focal adhesions to the cell surface, where it co-localizes with Robo4. To test this hypothesis, we analysed the subcellular distribution of paxillin and Robo4 in the absence and presence of Slit2. Because Slit2 blocks cell spreading and prevents clear visualization of the plasma membrane, we performed these experiments in endothelial cells pre-spread on fibronectin. In the absence of Slit2, paxillin was found almost exclusively in focal adhesions, whereas Robo4 was localized to the cell surface (Supplementary Information, Fig. S3c, top panel). In the presence of Slit2, however, a significant portion of paxillin appeared at the cell surface, co-localized with Robo4; this alteration in localization was coincident with a reduction of paxillin in focal adhesions (Supplementary Information, Fig. S3c, middle panel).

Thus far, our experiments had used model cell systems to decipher signal transduction downstream of Robo4. To determine whether this pathway is important for Robo4 function in primary cells, we first subjected human endothelial cells to haptotaxis assays and, analogous to the model cell system, Slit2 blocked fibronectin-driven cell motility (Fig. 4a). We also performed spreading assays on coverslips coated with the 9-11 fragment of fibronectin (a ligand for $\alpha 5 \beta 1$ integrin) and Slit2, and again found that Slit2 suppressed cellular protrusive activity stimulated by integrin ligation (Fig. 4b). To analyse a potential role for GIT1 in Slit2dependent inhibition of cell protrusion, we pre-treated endothelial cells with a small-molecule inhibitor of Arf-GAPs, Qs11 (ref. 21), and subjected them to spreading assays on the 9-11 fibronectin fragment and Slit2. Arf-GAP-inhibition prevented the reduction in cell area elicited by Slit2 (Fig. 4b). 
Next, we determined the requirement for paxillin binding to Robo4 in Slit2-dependent inhibition of cell migration. We depleted endogenous Robo4 in human endothelial cells using siRNA, and reconstituted the receptor using adenoviral vectors expressing full-length murine Robo4, Robo4 ED-TM or Robo4 $\triangle \mathrm{PIM}$, and subjected these cells to migration assays in the absence and presence of Slit2. The migration of control cells and Robo4-knockdown cells reconstituted with full-length Robo4 was inhibited by Slit2, but Slit2 had no effect on Robo4knockdown cells reconstituted with either Robo4 ED-TM or Robo4 $\triangle$ PIM (Fig. 4c).

These cell biological data suggest that Slit2-Robo4 signalling in endothelial cells blocks Rac and Arf6 activation in response to integrin ligation. To test this idea, we plated endothelial cells on fibronectin in the absence and presence of Slit2, and analysed Rac-GTP and Arf6-GTP levels. Consistent with results from the model cell systems, Slit2 blocked the fibronectininduced increase in Rac-GTP (Fig. 4d) and Arf6-GTP levels (Fig. 4e). In addition to fibronectin, the angiogenic and permeability-inducing factor VEGF-165 exists in vivo as an extracellular matrix component, and has been suggested to stimulate Arf6 activation ${ }^{22}$. Consequently, we plated endothelial cells on VEGF-165 in the absence and presence of Slit2, and analysed Arf6-GTP levels. Whereas VEGF-165 induced Arf6 activation, addition of Slit2 prevented this stimulatory event (Fig. 4f).

We previously demonstrated that the effect of Slit2 on endothelial cell biology is Robo4dependent ${ }^{6}$. To determine whether this relationship exists for the biochemical regulation of Arf6, we plated endothelial cells from $R o b o 4^{+/+}$and $R o b o 4^{\mathrm{AP} / \mathrm{AP}}$ mice ${ }^{6}$ on fibronectin, in the absence and presence of Slit2, and analysed Arf6-GTP levels. Slit2 decreased activated Arf6 in $R_{o b o} 4^{+/+}$, but notRobo4 AP/AP, endothelial cells (Supplementary Information, Fig. S4).

In addition to Arf6 and Rac, Rho and Cdc42 have known roles in cellular protrusion ${ }^{18}$. To gain insight into the regulation of these small GTPases by Slit2, we plated endothelial cells on fibronectin in the absence and presence of Slit2, and analysed Rho-GTP and Cdc42-GTP levels. Whereas Rho activation was unaltered by Slit2, Cdc 42 activation was significantly reduced (Supplementary Information, Fig. S5a, b). This latter effect was surprising given that Robo4 does not interact with the Robo1-binding protein srGAP1, a known Cdc42 GTPaseactivating protein (Supplementary Information, Fig. S5c).

Recently, we showed that Robo4 mediates Slit2-dependent inhibition of neovascular tuft formation and endothelial hyperpermeability ${ }^{6}$, processes that are initiated and perpetuated by endothelial integrins and growth factor receptors. The ability of Slit2 to block Arf6 activation in response to fibronectin and VEGF-165 (which are ligands for these angiogenic and permeability-inducing receptors) led us to speculate that Arf6 is a critical nexus in signalling pathways regulating pathologic angiogenesis and vascular leak ${ }^{22}$. To test this hypothesis, we used a recently developed small-molecule inhibitor of cytohesin Arf-GEFs, SecinH3, which blocks insulin-induced Arf6 signalling23. SecinH3, but not DMSO, prevented both VEGFinduced Arf6 activation and VEGF-induced endothelial cell migration (Fig. 5a, b).

Furthermore, injection of SecinH3 into the eyes of wild-type mice inhibited neovascular tuft formation in oxygen-induced retinopathy (Fig. 5c, d) and choroidal neovascularization (Fig. 5e, f), as well as retinal hyperpermeability caused by VEGF-165 (Fig. 5g).

Cumulatively, these data demonstrate the importance of paxillin and GIT1 in mediating Slit2Robo4-dependent inhibition of endothelialcell protrusive activity, and suggest that blocking activation of Arf6 is a potential therapy for human diseases characterized by pathologic angiogenesis and vascular leak (Fig. 5h). 


\section{METHODS}

\section{Reagents}

HEK 293 and COS-7 cells, and all IMAGE clones were from ATCC. SP6 and T7 Message Machine kits were from Ambion. Glutathione-Sepharose 4B, parental pGEX-4T1 and ECL PLUS were from Amersham-Pharmacia. Human fibronectin was from Biomedical Technologies and Invitrogen. Coomassie blue and PVDF (polyvinyldifluoride) were from BioRad. Yeast two-hybrid plasmids and reagents were from Clontech. Costar Transwells and Amicon Ultra-15 Concentrator Columns were from Fisher. FBS was from Hyclone. Anti-V5 antibody, DAPI, DMEM, Lipofectamine 2000, Penicillin-Streptomycin, Prolong Gold, Superscript III kit, Trizol and TrypLE Express were from Invitrogen. Goat Anti-Mouse-HRP and Goat Anti-Rabbit-HRP secondary antibodies were from Jackson ImmunoResearch. 3D Blind Deconvolution algorithm of AutoQuantX was from Media Cybernetics. HMVEC, HUVEC, EBM-2 and bullet kits were from Lonza. Alexa564-Phalloidin, Anti-GFP and Goat Anti-Rabbit Alex488 were from Molecular Probes. Rosetta2 Escherichia coli was from Novagen. Low melt agarose was from NuSieve. $\mathrm{T} 7$ in vitro transcription/translation kit was form Promega. Anti-HA affinity matrix, Fugene6 and protease inhibitor cocktail were from Roche. Arf6 antibody and normal Rat IgG-agarose conjugate was from Santa Cruz. Anti-Flag M2, phosphatase inhibitor cocktails, soybean trypsin inhibitor and fatty acid-free bovine serum albumin (BSA) were from Sigma. Nikon Eclipse TE2000-U microscope was from Semrock. Quick change site-directed mutagenesis kit was from Stratagene. Oligonucleotides for PCR were from the University of Utah Core Facility.

\section{Molecular biology}

The Robo4-HA, Slit2-Myc-His and chicken paxillin plasmids have been described previously $2,7,20$. Robo4-NH2 was amplified from Robo4-HA and cloned into EcoRV/NotI of pcDNA3-HA. Robo4-COOH was amplified from Robo4-HA by overlap-extension PCR and cloned into EcoRV/NotI of pcDNA-HA. The N-terminal half of the human Robo4 cytoplasmic tail (amio-acids 465-723) was amplified by PCR and cloned into (EcoRI/BamHI) of pGBKT7. Murine Robo4 fragments were amplified by PCR and cloned into BamHI/EcoRI of pGEX-4T1. Murine Hic-5, Mena and paxillin (including deletions) were amplified from IMAGE clones by PCR and cloned into EcoRV/NotI of pcDNA3-V5. GST-Robo4 $\Delta$ PIM and full-length Robo4 $\triangle \mathrm{PIM}$ were generated by site-directed mutagenesis of relevant wild-type constructs using Quick Change. The integrity of all constructs was verified by sequencing at the University of Utah Core Facility.

\section{Cell culture}

HEK 293 and COS-7 cells were cultured in DMEM supplemented with 10\% FBS and 1\% penicillin/streptomycin. Human microvascular endothelial cells (HMVEC) were cultured in EGM-2 MV and human umbilical vein endothelial cells (HUVEC) were cultured in EGM-2 supplemented with 10\% FBS. Cells were used between passages 2 and 5 .

\section{Transfection}

HEK293 and COS-7 cells were transfected with Fugene6 or Lipofectamine 2000 according to the manufacturer's protocol.

\section{Preparation of concentrated recombinant Slit2 protein}

Slit2 and mock were prepared as described previously ${ }^{6}$. Mock is sham preparation of Slit2, and was used as a control in all experiments analysing the effect of Slit2. 


\section{Haptotaxis migration assay}

Cells were removed from tissue culture dishes with TrypLE Express, washed once with $0.1 \%$ trypsin inhibitor, $0.2 \%$ fatty acidfree BSA in DMEM or EBM-2, and twice with $0.2 \%$ BSA in the relevant media. The washed cells were counted, resuspended, and $1.5 \times 10^{5}$ (HEK) or 0.2 $\times 10^{5}$ (HMVEC) cells were loaded into the upper chamber of $12-\mu \mathrm{m}$ (HEK) or 8- $\mu \mathrm{m}$ (HMVEC) Costar transwells pre-coated on the lower surface with fibronectin $\left(5 \mu \mathrm{g} \mathrm{ml}^{-1}\right)$. The effect of Slit2 on haptotaxis was analysed by co-coating with Slit2 $\left(5 \mu \mathrm{g} \mathrm{ml}^{-1}\right)$ or an equivalent amount of mock. After $6 \mathrm{~h}$ (HEK) or $3 \mathrm{~h}$ (HMVEC), cells on the upper surface of the transwell were removed with a cotton swab. The cells on the lower surface were fixed with $4 \%$ formaldehyde, washed three times with PBS and mounted in Vectashield with DAPI. The number of GFPpositive (HEK) and DAPI-positive (HMVEC) cells on the lower surface was determined by counting six 10× fields on an inverted fluorescence microscope. The number of migrated cells on fibronectin/mock-coated membranes was set at $100 \%$. Experiments were performed at least three times in triplicate.

\section{Yeast two hybrid assay}

pGBKT7::hRobo4 465-723 was transformed into the yeast strain PJ694A, creating PJ694ARobo4. A human aortic cDNA library was cloned into the prey plasmid pACT2 and then transformed into PJ694A-Robo4. Co-transformed yeast strains were plated onto SD-Leu-Trp (-LT) to analyse transformation efficiency and SD-Leu-Trp-His-Ade (-LTHA) to identify putative interacting proteins. Yeast strains competent to grow on SD-LTHA were then tested for expression of $\beta$-galactosidase by a filter lift assay. Prey plasmids were isolated from yeast strains capable of growing on SD-LTHA and expressing $\beta$-galactosidase, and sequenced at the University of Utah Core Facility.

\section{Immunoprecipitation}

Cell lysates were prepared in $50 \mathrm{mM}$ Tris-Cl at $\mathrm{pH} 7.4,50 \mathrm{mM} \mathrm{NaCl}, 1 \mathrm{mM}$ DTT, $0.5 \%$ Triton $\mathrm{X}-100$, phosphatase and protease inhibitors, centrifuged at 16,000 $\mathrm{g}$ for $20 \mathrm{~min}$, cleared with normal IgG coupled to agarose beads for $60 \mathrm{~min}$, and incubated for $2 \mathrm{~h}$ at $4{ }^{\circ} \mathrm{C}$ with relevant antibodies coupled to agarose beads. The precipitates were washed extensively in lysis buffer and resuspended in $2 \times$ sample buffer $(125 \mathrm{mM}$ Tris-Cl at pH 6.8, 4\% SDS, 20\% Glycerol, $0.04 \%$ bromophenol blue and 1.4 M 2-mercaptoethanol).

\section{GST pulldown assay}

Rosetta2 E. coli cells harbouring pGEX-4T1::mRobo4 were grown to an optical density at 600 $\mathrm{nm}\left(\mathrm{OD}_{600}\right)$ of 0.6 and induced with IPTG $(0.3 \mathrm{mM})$. After $3-4 \mathrm{~h}$ at $30^{\circ} \mathrm{C}$, cells were centrifuged at 2,800 $\mathrm{g}$ for $5 \mathrm{~min}$, cells were lysed by sonication in $20 \mathrm{mM}$ Tris-Cl at $\mathrm{pH} 7.4,1 \%$ Triton $\mathrm{X}-100$, lysozyme $\left(1 \mu \mathrm{g} \mathrm{ml}^{-1}\right)$, DTT $(1 \mathrm{mM})$ and protease inhibitors. The GST fusion proteins were captured on glutathione-Sepharose $4 \mathrm{~B}$, washed once with lysis buffer without lysozyme and then twice with binding/wash buffer (50 $\mathrm{mM}$ Tris-Cl at pH 7.4, $150 \mathrm{mM} \mathrm{NaCl}, 1 \mathrm{mM}$ DTT, $1 \%$ Triton X-100, 0.1\% BSA and protease inhibitors). The GST fusion proteins were incubated with purified recombinant paxillin $(60 \mathrm{nM})$ overnight at $4{ }^{\circ} \mathrm{C}$, washed extensively in binding/ wash buffer, and resuspended in $2 \times$ sample buffer.

\section{Immunoblotting}

Protein samples were incubated for $2 \mathrm{~min}$ at $100^{\circ} \mathrm{C}$, separated by SDS-PAGE and transferred to a PVDF membrane. PVDF membranes were incubated with 5\% non-fat dry milk in PBS + $0.1 \%$ Tween 20 (PBST; PBST-M) for $60 \mathrm{~min}$ at $25^{\circ} \mathrm{C}$. Blocked membranes were incubated with primary antibodies (against Arf6, Cdc42, Flag M2, Hic-5, paxillin, Rac and Rho) in PBST$\mathrm{M}$ for $60 \mathrm{~min}$ at $25^{\circ} \mathrm{C}$, or overnight at $4{ }^{\circ} \mathrm{C}$. Membranes were washed for $10 \mathrm{~min}$, three times, in PBST and then incubated with a secondary antibody (goat anti-mouse or goat anti-rabbit 
horseradish peroxidase) for $60 \mathrm{~min}$ at $25^{\circ} \mathrm{C}$. Membranes were washed for $10 \mathrm{~min}$, three times, in PBST and visualized with ECL PLUS. Primary antibodies were used at the following dilutions: antibodies against GTPases, 1:1,000; Flag, 1:1,000; Hic-5, 1:500 and paxillin, $1: 10,000$.

\section{In vitro transcription/translation}

Mena-V5 was synthesized with the T7 Quick Coupled in vitro Transcription/Translation system according to the manufacturer's protocol.

\section{Spreading assay}

Cell spreading was analysed as described previously ${ }^{20}$. The cell area from at least 150 cells, from three independent experiments, was measured using ImageJ (NIH).

\section{Depletion and reconstitution of paxillin}

HEK 293 cells were transfected with $100 \mathrm{nM}$ of siRNA duplexes (5'CCCUGACGAAAGAGAAGCCUAUU-3' and 5'UAGGCUUCUCUUUCGUCAGGGUU-3') using Lipofectamine 2000, according to the manufacturer's instructions. At $48 \mathrm{~h}$ after transfection, cells were processed for biochemical analysis or cell spreading assays. Paxillin reconstitution was accomplished by transfection with an expression vector encoding chicken paxillin, which has the nucleotide sequence 5'CCCCTACAAAAGAAAAACCAA-3' within the siRNA target site. Knockdown and reconstitution were analysed by immunoblotting with paxillin antibodies, and quantified by densitometry.

\section{GTPase activation assays}

GTPase activation was analysed as described previously ${ }^{20}$. Experiments were performed at least three times.

\section{Adenoviral preparation}

Murine full-length Robo4, Robo4 $\triangle \mathrm{PIM}$, and Robo4 ED-TM were subcloned into pShuttleIRES-hrGFP-2 (Stratagene). Independently, these vectors were used with pAdEasy to cotransform BJ5183 cells (Stratagene). Recombinant plasmids were linearized by PacI digestion and transfected into 293 cells for packaging. The adenovirus was then amplified and titered for plaque forming units (PFU).

\section{Depletion and reconstitution of Robo4}

huRobo4 siRNA duplex (Hs_ROBO4_1_HP \#1919431, Qiagen) or, alternatively, equimolar AllStars Negative Control siRNA (\#1027280, Qiagen) was diluted to $480 \mathrm{nM}$ in OptiMEM (Invitrogen). To form transfection complexes, siRNAs were premixed for 5-15 minutes at room temperature with HiPerfect Transfection Reagent (70 $\mu$; Qiagen) and added to human microvascular endothelial cells. After an overnight incubation, cells were re-transfected with siRNA according to the same protocol. Adenoviral constructs were also added at a concentration of $1 \times 10^{6} \mathrm{PFU} \mathrm{ml}{ }^{-1}$ and allowed to incubate overnight.

\section{Immunofluorescence imaging}

Glass coverslips were coated sequentially with human plasma fibronectin $\left(5 \mu \mathrm{g} \mathrm{ml}^{-1}\right)$ and Slit2 or Mock $\left(5 \mu \mathrm{g} \mathrm{ml}^{-1}\right)$, and blocked with $1 \%$ BSA. HEK Robo4 and bovine aortic endothelial (BAE) cells were then adhered for $30 \mathrm{~min}$ in serum-free DMEM supplemented with $1 \%$ BSA, and fixed with $3.7 \%$ formaldehyde in PBS. Cells were permeabilized in $0.1 \%$ Triton X-100 for $5 \mathrm{~min}$, blocked and stained for Paxillin using either a rabbit polyclonal antibody or a mouse 
monoclonal antibody and counterstained with a FITC-conjugated secondary antibody. When indicated, cells were co-stained for F-actin with rhodamine-phalloidin. Coverslips were subsequently mounted in Prolong Gold antifade reagent on slides. Epi-fluorescent images of cells were acquired with a $60 \times$ oil immersion objective on a Nikon Eclipse TE2000-U microscope. Images as shown are maximal projections of deconvolved images that were acquired at $0.1-\mu \mathrm{m} z$-section intervals. Images were deconvolved using the 3D Blind Deconvolution algorithm of AutoQuantX. Additional post-acquisition processing of images was performed using ImageJ and Adobe Photoshop.

\section{Retinal permeability}

Retinal permeability was assessed as described previously15.Data are presented as mean \pm s.e.m. for six wild-type mice.

\section{Oxygen-induced retinopathy}

Oxygen-induced retinopathy was induced as described previously ${ }^{15}$. Data are presented as mean \pm s.e.m. for 14 wild-type mice.

\section{Laser-induced choroidal neovascularisation}

Laser-induced choroidal neovascularization was induced as described previously ${ }^{15}$. Data are presented as mean \pm s.e.m. for at least 15 wild-type mice.

\section{Statistical analysis}

Student's $t$-test or ANOVA was used to determine statistical significance, where appropriate. All $P$ values were derived from at least three independent experiments.

\section{Supplementary Material}

Refer to Web version on PubMed Central for supplementary material.

\section{Acknowledgments}

We thank J. Wythe for critical reading of the manuscript, and D. Lim for expert graphical assistance. J. Bonafacino provided the GST-GGA3 expression plasmid. This work was funded by grants from the H.A. and Edna Benning Foundation, the Juvenile Diabetes Research Foundation, the American Heart Association, the Burroughs Wellcome Fund and the Department of Defense (D.Y.L.); the National Heart Lung and Blood Institute (D.Y.L. and M.H.G.); the National Eye Institute (K.Z.); the Deutsche Forschungsgemeinschaft SFB 704 (M.F.); the National Institute of Arthritis and Musculoskeletal and Skin Diseases (M.H.G.); the National Institute of Allergy and Disease AI065357 (D.Y.L.) and by the US National Institutes of Health, Ruth L. Kirschstein National Research Service Award (N.R.L.) and training grant T32-GM007464 (A.C.C.).

\section{References}

1. Seeger M, Tear G, Ferres-Marco D, Goodman CS. Mutations affecting growth cone guidance in Drosophila: genes necessary for guidance toward or away from the midline. Neuron 1993;10:409426. [PubMed: 8461134]

2. Brose K, et al. Slit proteins bind Robo receptors and have an evolutionarily conserved role in repulsive axon guidance. Cell 1999;96:795-806. [PubMed: 10102268]

3. Kidd T, Bland KS, Goodman CS. Slit is the midline repellent for the robo receptor in Drosophila. Cell 1999;96:785-794. [PubMed: 10102267]

4. Kidd T, et al. Roundabout controls axon crossing of the CNS midline and defines a novel subfamily of evolutionarily conserved guidance receptors. Cell 1998;92:205-215. [PubMed: 9458045]

5. Li HS, et al. Vertebrate slit, a secreted ligand for the transmembrane protein roundabout, is a repellent for olfactory bulb axons. Cell 1999;96:807-818. [PubMed: 10102269] 
6. Jones CA, et al. Robo4 stabilizes the vascular network by inhibiting pathologic angiogenesis and endothelial hyperpermeability. Nature Med 2008;14:448-453. [PubMed: 18345009]

7. Park KW, et al. Robo4 is a vascular-specific receptor that inhibits endothelial migration. Dev. Biol 2003;261:251-267. [PubMed: 12941633]

8. Seth $\mathrm{P}$, et al. Magic roundabout, a tumor endothelial marker: expression and signaling. Biochem. Biophys. Res. Comm 2005;332:533-541. [PubMed: 15894287]

9. Ridley AJ, et al. Cell migration: integrating signals from front to back. Science 2003;302:1704-1709. [PubMed: 14657486]

10. Pollard TD, Borisy GG. Cellular motility driven by assembly and disassembly of actin filaments. Cell 2003;112:453-465. [PubMed: 12600310]

11. Etienne-Manneville S, Hall A. Rho GTPases in cell biology. Nature 2002;420:629-635. [PubMed: 12478284]

12. Turner CE. Paxillin and focal adhesion signalling. Nature cell Biol 2000;2:E231-236. [PubMed: 11146675]

13. Yuminamochi T, et al. Expression of the LIM proteins paxillin and Hic-5 in human tissues. J. Histochem. Cytochem 2003;51:513-521. [PubMed: 12642630]

14. Hohenester E, Hussain S, Howitt JA. Interaction of the guidance molecule Slit with cellular receptors. Biochem. Soc. Trans 2006;34:418-421. [PubMed: 16709176]

15. Jones CA, et al. Robo4 stabilizes the vascular network by inhibiting pathologic angiogenesis and endothelial hyperpermeability. Nature Med. 2008

16. Bashaw GJ, Kidd T, Murray D, Pawson T, Goodman CS. Repulsive axon guidance: Abelson and Enabled play opposing roles downstream of the roundabout receptor. Cell 2000;101:703-715. [PubMed: 10892742]

17. Turner CE. Paxillin interactions. J. Cell Sci 2000;113(Pt 23):4139-4140. [PubMed: 11069756]

18. Nobes CD, Hall A. Rho, rac, and cdc42 GTPases regulate the assembly of multimolecular focal complexes associated with actin stress fibers, lamellipodia, and filopodia. Cell 1995;81:53-62. [PubMed: 7536630]

19. Nobes CD, Hall A. Rho GTPases control polarity, protrusion, and adhesion during cell movement. J. Cell Biol 1999;144:1235-1244. [PubMed: 10087266]

20. Nishiya N, Kiosses WB, Han J, Ginsberg MH. An alpha4 integrin-paxillin-ArfGAP complex restricts Rac activation to the leading edge of migrating cells. Nature Cell Biol 2005;7:343-352. [PubMed: 15793570]

21. Zhang Q, et al. Small-molecule synergist of the Wnt/ -catenin signaling pathway. Proceedings of the National Academy of Sciences of the United States of America 2007;104:7444-7448. [PubMed: 17460038]

22. Ikeda S, et al. Novel role of ARF6 in vascular endothelial growth factor-induced signaling and angiogenesis. Circ. Res 2005;96:467-475. [PubMed: 15692085]

23. Hafner M, et al. Inhibition of cytohesins by SecinH3 leads to hepatic insulin resistance. Nature 2006;444:941-944. [PubMed: 17167487] 

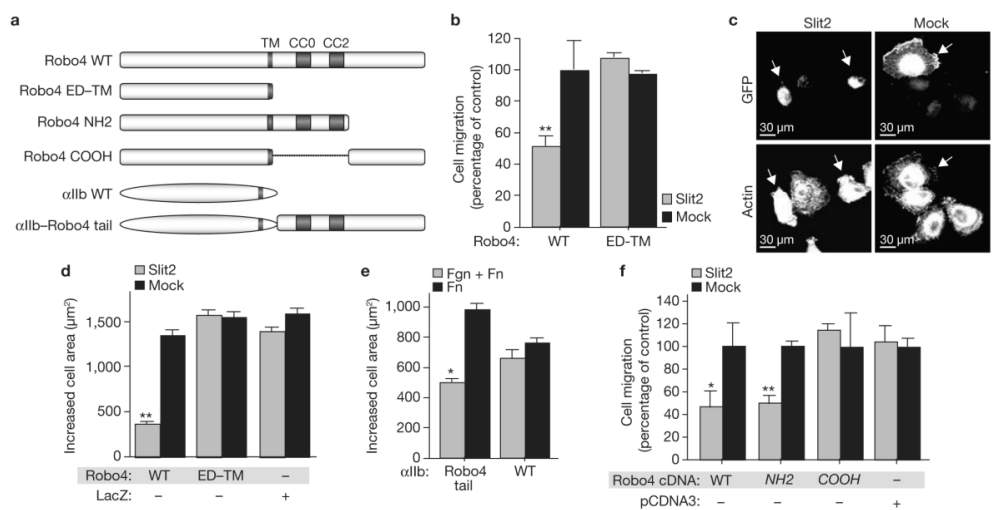

Figure 1.

The Robo4 cytoplasmic tail suppresses fibronectin-induced protrusive activity. (a) Schematic representation of cDNA constructs used in migration and spreading assays. TM,

transmembrane domain. $\mathrm{CC} 0$ and $\mathrm{CC} 2$ are conserved cytoplasmic signalling motifs found in Robo family members. (b-d, f) HEK 293 cells expressing GFP and the indicated constructs were subjected to haptotaxis migration assays (b, f) or spreading assays (c, d) on fibronectin and mock or Slit2. Arrows indicate GFPpositive cells. (e) CHO-K1 cells stably expressing $\alpha I I b$ integrin or $\alpha$ IIb integrin-Robo4 cytoplasmic tail were subjected to spreading assays on fibronectin (Fn) or fibronectin and fibrinogen (Fn + Fgn). Cell area was determined using ImageJ software. For migration assays, GFP-positive cells on the underside of the filter were counted and migration on fibronectin or mock membranes was set at $100 \%(\mathrm{n}=3$, in triplicate). For spreading assays, the area of GFP-positive cells was determined using ImageJ ( $n=3,150$ cells per experiment). Expression of Robo4 constructs was verified by immunoblotting (data not shown). ${ }^{*} P<0.05 ; * * P<0.005$. Error bars indicate the mean \pm s.e.m. WT, wild type. Mock, a sham preparation of Slit2. $\alpha$ IIb-Robo4, $\alpha$ IIb integrin-Robo4. pCDNA3, empty vector. 


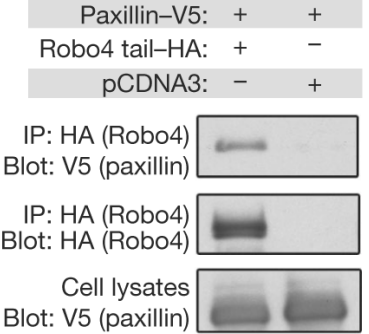

b

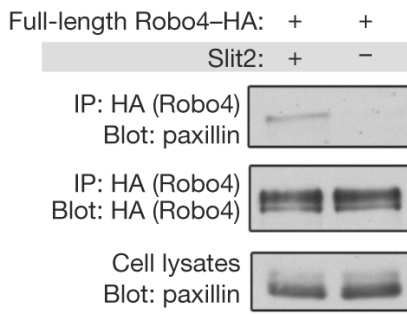

e

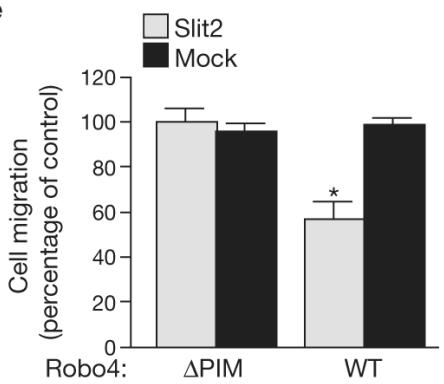

h

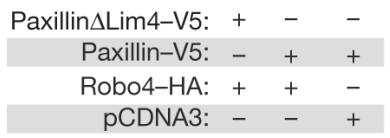

IP: HA (Robo4) Blot: V5 (paxillin)

IP: HA (Robo4) Blot: HA (Robo4)

Cell lysates

Blot: V5 (paxillin)

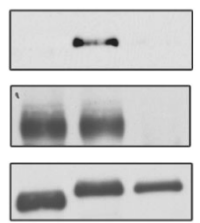

c

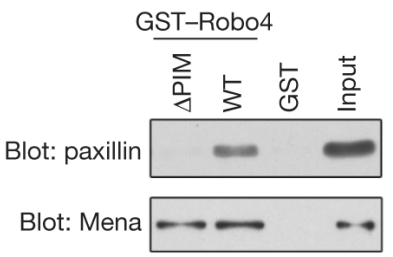

f

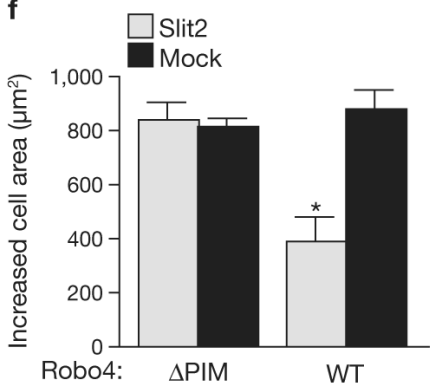

i

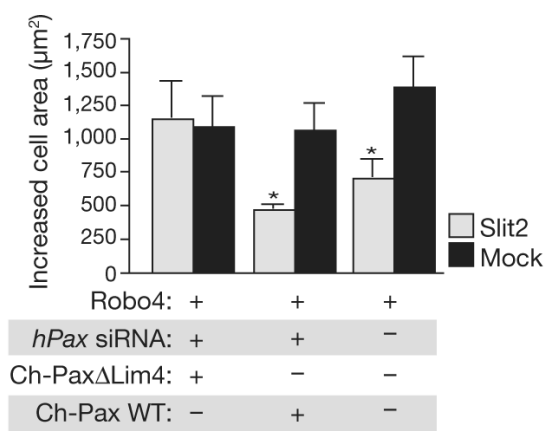

Figure 2.

Robo4-paxillin interaction is required for Slit2-dependent inhibition of protruive activity. (a) Lysates from HEK 293 cells expressing paxillin-V5 and Robo4 cytoplasmic tail-HA or empty vector (pcDNA3) were immunoprecipitated with HA antibodies and immunoblotted with V5 antibodies. (b) Lysates from Slit2-stimulated HEK 293 cells expressing full length Robo4-HA were immunoprecipitated with HA antibodies and immunoblotted with paxillin antibodies. (c) Purified GST-Robo4 wild type or GST-Robo4 $\triangle$ PIM were incubated with recombinant purified paxillin or in vitro transcribed/translated Mena-V5, precipitated with glutathione agarose, and immunoblotted with paxillin or V5 antibodies. (d) Lysates from HEK293 cells expressing wild-type Robo 4 cytoplasmic tail-HA, Robo4 $\Delta$ PIM cytoplasmic tail-HA or empty vector (pcDNA3) were immunoprecipitated with HA antibodies and immunoblotted with paxillin antibodies. (e, f) HEK 293 cells expressing GFP and the indicated constructs were subjected to haptotaxis migration assays (e) or spreading assays (f) on fibronectin and mock or Slit2. (g) Lysates from HEK 293 cells expressing Robo4 cytoplasmic tail-HA and either wild-type paxillin-V5 or paxillin $\Delta$ Lim4-V5 were immunoprecipitated with HA antibodies and immunoblotted with V5 antibodies. (h) Endogenous paxillin was depleted in HEK 293 cells using siRNA and reconstituted with wild-type chicken (Ch-) paxillin or Chpaxillin $\Delta$ Lim4. Lysates were immunoblotted with paxillin antibodies. (i)Depleted/reconstituted HEK 293 cells were subjected to spreading assays on fibronectin and mock or Slit2. In migration assays, GFPpositive cells on the underside of the filter were counted and migration on fibronectin/mock membranes was set at $100 \%(\mathrm{n}=3$, in triplicate). In spreading assays, the area of GFP-positive cells was determined using ImageJ software ( $n=3,150$ cells per experiment). Expression of 
Robo4 constructs was verified by immunoblotting (data not shown). $* P<0.05$. Error bars indicate the mean \pm s.e.m. Full scans of blots are shown in Supplementary Information, Fig. S6. WT, wild type. Mock, sham preparation of Slit2. 
a

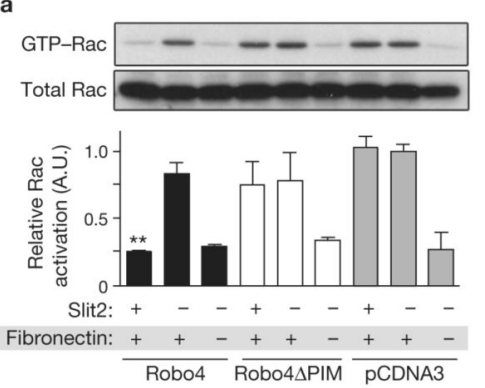

b

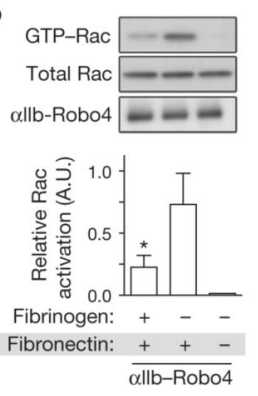

c

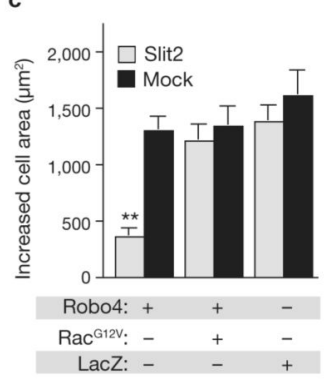

d
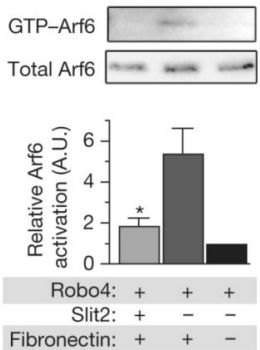
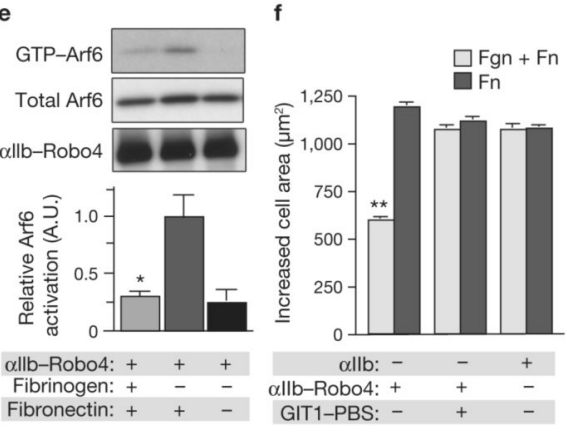

g

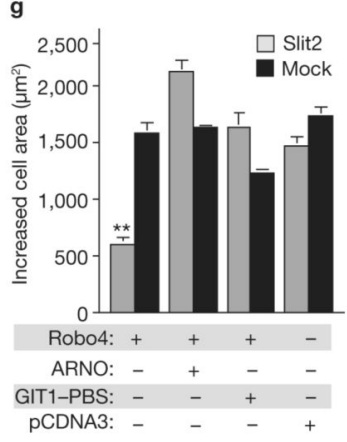

h

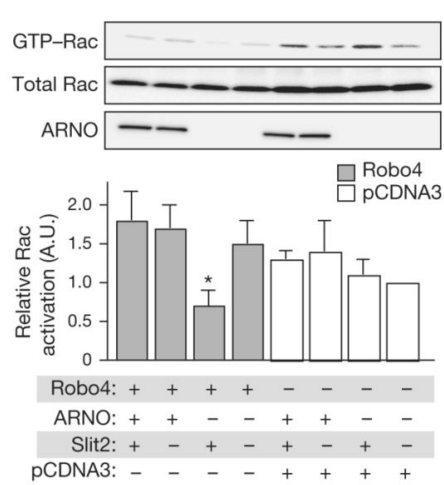

Figure 3.

obo4 blocks Rac-dependent protrusive activity through inhibition of Arf6. (a) Lysates from HEK 293 cells expressing the indicated constructs,plated on fibronectin and mock or Slit2, were precipitated with GST-PBD and immunblotted with Rac antibodies $(\mathrm{n}=3)$. (b) Lysates from CHO-K1 cells expressing $\alpha$ IIb-Robo4 were precipitated with GST-PBD and immunoblotted with Rac antibodies $(\mathrm{n}=3$ ). (c) HEK 293 cells expressing Robo4 and RacG12Vor LacZ were subjected to spreading assays on fibronectin and mock or Slit2.(d, e) Lysates from HEK 293 cells expressing full-length Robo4 (d) and CHO-K1 cells expressing aIIb integrin-Robo4 cytoplasmic tail (e), plated on the indicated matrix, were precipitated with GST-GGA3 and immunoblotted with Arf6 antibodies $(\mathrm{n}=3$ ). (f) CHO-K1 cells stably expressing $\alpha \mathrm{IIb}$ or $\alpha \mathrm{IIb}$ integrin-Robo4 cytoplasmic tail were co-transfected with GFP and either an empty vector or GIT1-PBS, and were subjected to spreading assays on the indicated matrix. (g) HEK 293 cells expressing GFP and the indicated constructs were subjected to spreading assays on fibronectin and Mock or Slit2. Expression of Robo4 and ARNO was verified by immunoblotting (data not shown). (h) Lysates from HEK 293 cells expressing the indicated constructs, plated on fibronectin and mock or Slit2, were precipitated with GSTPBD and immunoblotted with Rac antibodies $(n=3)$. For spreadingassays, the area of GFPpositive cells was determined using ImageJ software $(n=3,150$ cells per experiment). Expression of Robo4 constructs was verifiedby western blotting (data not shown). $* P<0.05$; $* * P<0.005$. Error bars xindicate the mean \pm s.e.m. Full scans of blots are shown in Supplementary Information, Fig. S7. aIIb-Robo4 indicates aIIb integrin-Robo4. 

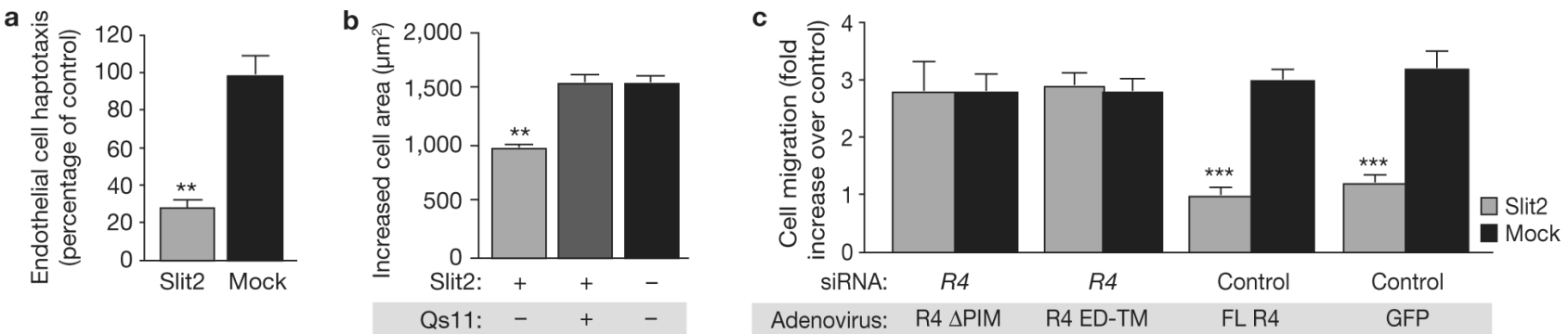

d
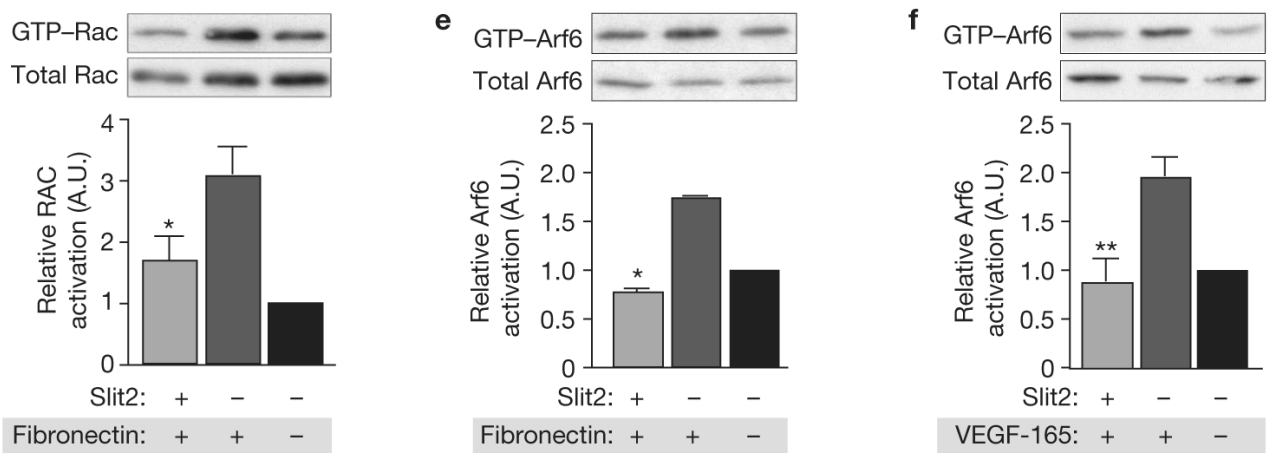

Figure 4.

Slit2-Robo4 signalling inhibits endothelial cell protrusive activity through paxillin and ArfGAPs. (a) Endothelial cells were subjected to haptotaxis migration assays on fibronectin and mock or Slit2. Cells on the underside of the filter were counted and migration on fibronectin/ mock membranes was set at $100 \%(\mathrm{n}=3$, in triplicate). (b) Endothelial cells were subjected to spreading assays on fibronectin and mock or Slit2, in the absence and presence of Qs11 (n $=3,150$ cells per experiment). Cell area was determined using ImageJ software. (c) Robo4 was depleted in endothelial cells using siRNA. Cells were then infected with an adenovirus expressing the indicated constructs, and were subjected to migration assays $(\mathrm{n}=5$, in triplicate). (d, e) Lysates from endothelial cells plated on fibronectin and either mock or Slit2, were precipitated with GST-PBD and immunoblotted with Rac antibodies (d) or GST-GGA3 and immunoblotted with Arf6 antibodies (e; $n=3$ in e and f). (f) Lysates from endothelial cells plated on VEGF-165 and mock or Slit2 were precipitated with GST-GGA3 and immunoblotted with Arf6 antibodies $(\mathrm{n}=3)$. $* P<0.05, * * P<0.005$ and $* * * P<0.0005$. Error bars indicate the mean \pm s.e.m. Full scans of blots are shown in Supplementary Information, Fig. S8. R4, Robo4. FL, full-length. 

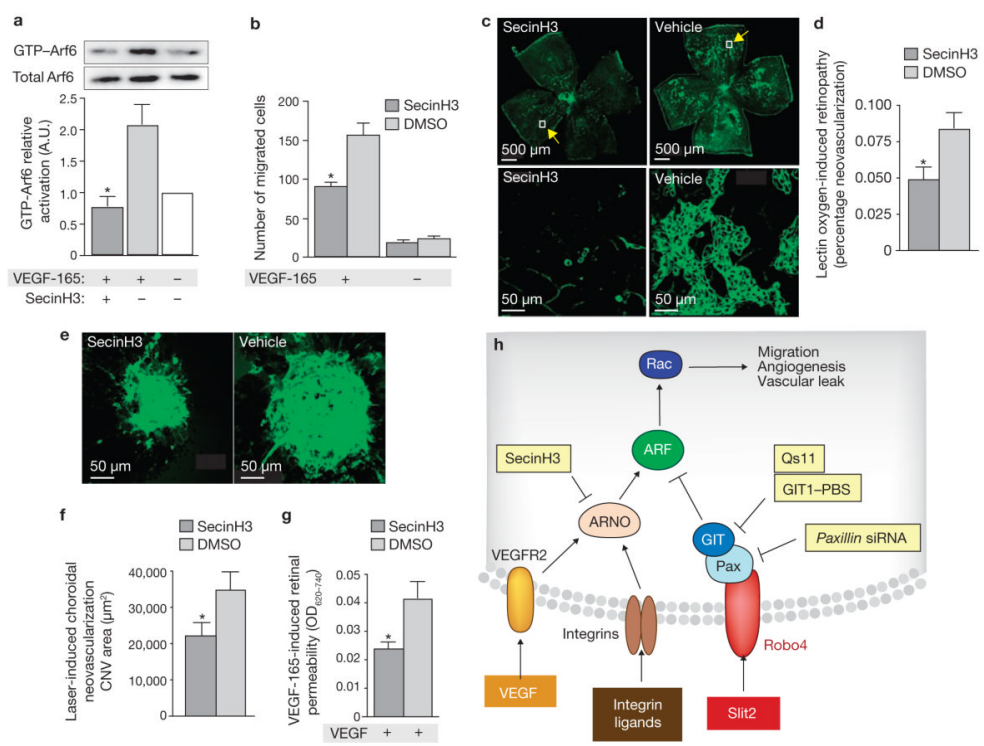

Figure 5.

SecinH3 blocks Arf6 activation and inhibits pathologic angiogenesis and endothelial hyperpermeability in animal models of vascular eye disease.(a) Lysates from endothelial cells, pre-treated with SecinH3 or DMSO and then stimulated with VEGF-165, were precipitated with GST-GGA3 and immunoblotted with Arf6 antibodies. (b) Endothelial cells, pre-treated with SecinH3 or DMSO, were subjected to migration assays using VEGF-165.(c, e, g) SecinH3 or DMSO were injected into contralateral eyes of wildtype mice, which were then subjected to oxygen-induced retinopathy (c), laser-induced choroidal neovascularization (e) or VEGF-165-induced retinal hyperpermeability (g). (c) Retinal flatmounts prepared from neonatal mice subjected to oxygen-induced retinopathy were stained with fluorescent isolectin and analysed by fluorescence microscopy. Top panels are low magnification images, and bottom panels, high magnification images of the outlined areas, which emphasize the pathologic neovascular tufts.(d) Quantification of pathologic neovascularization shown in $\mathrm{c}(\mathrm{n}$ = 14animals). (e) Choroidal flatmounts prepared from 2-3- month-old mice subjected to laserinduced choroidal neovascularization were stained with fluorescent isolectin and analysed by confocal microscopy. (f) Quantification of pathologic angiogenesis observed in e ( $\mathrm{n}=15$ animals). (g) Quantification of retinal permeability following intravitreal injection of VEGF-165 ( $\mathrm{n}=6$ animals). Vehicle is DMSO. $* P<0.05$. Error bars indicate the mean \pm s.e.m. (h) VEGFR and integrin signalling promote sequential activation of Arf6 and Rac, leading to cellular protrusive activity. Concomitant initiation of Robo4-signalling by Slit2 stimulates recruitment of a paxillin-Arf-GAP complex to the cytoplasmic tail of the receptor, which causes local inactivation of Arf6and Rac, thereby blocking the protrusive activity necessary for angiogenesis and vascular leak. Full scans of blots are shown in SupplementaryInformation, Fig. S9. CNV, choroidal neovascularization. 